\title{
Color Confinement in QCD due to Topological Defects
}

\author{
Kei-Ichi Kondo* \\ ${ }^{*}$ Department of Physics, Faculty of Science, Chiba University, Chiba 263-8522, Japan ${ }^{1}$
}

\begin{abstract}
We outline a derivation of area law of the Wilson loop in SU(N) YangMills theory in the maximal Abelian gauge. This is based on a new version of nonAbelian Stokes theorem and the novel reformulation of the Yang-Mills theory. Abelian dominance and monopole dominance of the string tension in SU(N) QCD are immediate consequences of this derivation.
\end{abstract}

\section{INTRODUCTION}

In the dual superconductor picture [1] of quark confinement, the magnetic monopoles give the dominant contribution to the area law of the Wilson loop or the string tension. Based on 't Hooft argument [2], the partial gauge fixing $G \rightarrow H$ realizes the magnetic monopole in Yang-Mills gauge theory in the absence of elementary scalar field. In the conventional approach based on the maximal Abelian (MA) gauge, the residual gauge group was chosen to be the maximal torus subgroup $H=U(1)^{N-1}$ for $G=S U(N)$. This choice immediately determines the type of magnetic monopoles. We re-examine this issue. We learn that the magnetic monopole which is responsible for area law of the Wilson loop is determined by the maximal stability group $\tilde{H}$ rather than the residual gauge group $H$. This is a new feature appeared in $S U(N), N \geq 3$, which was overlooked so far in the lattice community as far as I know. Indeed, this situation occurs only for $S U(N), N \geq 3$. Therefore, we must distinguish the maximal stability group $\tilde{H}$ from the residual gauge group $H$. In general, the maximal stability group $\tilde{H}$ is larger than the maximal torus subgroup, $H=U(1)^{N-1} \subset \tilde{H}$. So, the coset $G / \tilde{H} \subset G / H$. We derive area law of the Wilson loop in $S U(N)$ Yang-Mills theory in the MA gauge. This is performed based on the results of a series of works [3-8].

1) Talk presented at New Directions in Quantum Chromodynamics, June 21-25, 1999, Kyungju, Korea. This work is supported in part by the Grant-in-Aid for Scientific Research from the Ministry of Education, Science and Culture (No.10640249). 


\section{COHERENT STATE}

First of all, we construct the coherent state $|\xi, \Lambda\rangle$ corresponding to the coset representatives $\xi \in G / \tilde{H}$. We define the maximal stability subgroup (isotropy subgroup) $\tilde{H}$ as a subgroup of $G$ that consists of all the group elements $h$ that leave the highest-weight state $|\Lambda\rangle$ invariant up to a phase factor, i.e., $h|\Lambda\rangle=|\Lambda\rangle e^{i \phi(h)}, h \in$ $\tilde{H}$. The phase factor is unimportant in the following discussion because we consider the expectation value of any operators in the coherent state. The maximal stability subgroup $\tilde{H}$ includes the Cartan subgroup $H=U(1)^{r}$, i.e., $H \subset \tilde{H}$.

Let $H$ be the Cartan subgroup of $G$ and $\mathcal{H}$ be the Cartan subalgebra in $\mathcal{G}$. For every element $g \in G$, there is a unique decomposition of $g$ into a product of two group elements, $g=\xi h, \xi \in G / \tilde{H}, h \in \tilde{H}$, for $g \in G$. We can obtain a unique coset space $G / \tilde{H}$ for a given $|\Lambda\rangle$. The action of arbitrary group element $g \in G$ on $|\Lambda\rangle$ is given by $g|\Lambda\rangle=\xi h|\Lambda\rangle=\xi|\Lambda\rangle e^{i \phi(h)}$.

The coherent state is constructed as $|\xi, \Lambda\rangle=\xi|\Lambda\rangle$. This definition of the coherent state is in one-to-one correspondence with the coset space $G / \tilde{H}$ and the coherent states preserve all the algebraic and topological properties of the coset space $G / \tilde{H}$. If $\Gamma^{\Lambda}(\mathcal{G})$ is Hermitian, then $H_{i}^{\dagger}=H_{i}$, and $E_{\alpha}^{\dagger}=E_{-\alpha}$. Every group element $g \in G$ can be written as the exponential of a complex linear combination of diagonal operators $H_{i}$ and off-diagonal shift operators $E_{\alpha}$. Thus the coherent state is given by

$$
|\xi, \Lambda\rangle=\xi|\Lambda\rangle=\exp \left[\sum_{\beta^{\prime}}\left(\eta_{\beta} E_{\beta}-\bar{\eta}_{\beta} E_{-\beta}\right)\right]|\Lambda\rangle, \quad \eta_{\beta} \in \mathbf{C}
$$

where $|\Lambda\rangle$ is the highest-weight state $\left(H_{j}|\Lambda\rangle=\Lambda_{j}|\Lambda\rangle, E_{\alpha}|\Lambda\rangle=0\right.$ for $\alpha \in R_{+}$, $R_{+}\left(R_{-}\right)$is a subsystem of positive (negative) roots.) such that

(i) $|\Lambda\rangle$ is annihilated by all the (off-diagonal) shift-up operators $E_{\alpha}$ with $\alpha>0$, $E_{\alpha}|\Lambda\rangle=0(\alpha>0)$

(ii) $|\Lambda\rangle$ is mapped into itself by all diagonal operators $H_{i}, H_{i}|\Lambda\rangle=\Lambda_{i}|\Lambda\rangle$;

(iii) $|\Lambda\rangle$ is annihilated by some shift-down operators $E_{\alpha}$ with $\alpha<0$, not by other $E_{\beta}$ with $\beta<0$ : $E_{\alpha}|\Lambda\rangle=0$ (some $\left.\alpha<0\right) ; E_{\beta}|\Lambda\rangle=|\Lambda+\beta\rangle($ some $\beta<0$ ); and the sum $\sum_{\beta^{\prime}}$ is restricted to those shift operators $E_{\beta}$ which obey (iii).

The coherent states are normalized to unity, $\langle\xi, \Lambda \mid \xi, \Lambda\rangle=1$, but, are nonorthogonal, $\left\langle\xi^{\prime}, \Lambda \mid \xi, \Lambda\right\rangle \neq 0$. The coherent state spans the entire space $V^{\Lambda}$. By making use of the the group-invariant measure $d \mu(\xi)$ of $G$ which is appropriately normalized, we obtain $\int|\xi, \Lambda\rangle d \mu(\xi)\langle\xi, \Lambda|=I$, which shows that the coherent states are complete, but overcomplete. This resolution of identity is very important to obtain the path integral formula given below. The coherent states $|\xi, \Lambda\rangle$ are in one-to-one correspondence with the coset representatives $\xi \in G / \tilde{H},|\xi, \Lambda\rangle \leftrightarrow G / \tilde{H}$. In other words, $|\xi, \Lambda\rangle$ and $\xi \in G / \tilde{H}$ are topologically equivalent. 
For concreteness, we first focus on the SU(3) case. The highest weight $\Lambda$ of the representation specified by the Dynkin index $[m, n]$ ( $m, n$ : integers) can be written as $\vec{\Lambda}=m \vec{h}_{1}+n \vec{h}_{2}$ ( $m, n$ are non-negative integers for the highest weight) where $h_{1}, h_{2}$ are highest weights of two fundamental representations of SU(3) corresponding to $[1,0],[0,1]$ respectively, i.e., $\vec{h}_{1}=\left(\frac{1}{2}, \frac{1}{2 \sqrt{3}}\right), \quad \vec{h}_{2}=\left(\frac{1}{2}, \frac{-1}{2 \sqrt{3}}\right)$. Therefore, we obtain $\vec{\Lambda}=\left(\frac{m+n}{2}, \frac{m-n}{2 \sqrt{3}}\right)$. If $m n=0$, i.e., $m=0$ or $n=0$, the maximal stability group $\tilde{H}$ is given by $\tilde{H}=U(2)$ (case (I)). Such a degenerate case occurs when the highest-weight vector $\vec{\Lambda}$ is orthogonal to some root vectors. If $m n \neq 0$, i.e., $m \neq 0$ and $n \neq 0, H$ is the maximal torus group $\tilde{H}=U(1) \times U(1)$ (case (II)). This is a non-degenerate case. Therefore, for the highest weight $\Lambda$ in the case (I), the coset $G / \tilde{H}$ is given by

$$
S U(3) / U(2)=S U(3) /(S U(2) \times U(1))=C P^{2},
$$

whereas in the case (II)

$$
S U(3) /(U(1) \times U(1))=F_{2} .
$$

Here, $C P^{n}$ is the complex projective space and $F_{n}$ is the flag space. Therefore, the two fundamental representations belong to the case (I), so the maximal stability group is $U(2)$, rather than the maximal torus group $U(1) \times U(1)$.

\section{NON-ABELIAN STOKES THEOREM}

We have derived a new version of non-Abelian Stokes theorem (NAST) $[9,10]$. For the non-Abelian Wilson loop defined by the trace of the path-ordered exponent along the closed loop $C$,

$$
W^{C}[\mathcal{A}]:=\operatorname{tr}\left[\mathcal{P} \exp \left(i g \oint_{C} \mathcal{A}\right)\right],
$$

with $\mathcal{A}$ being the connection one-form, $\mathcal{A}(x)=\mathcal{A}_{\mu}^{A}(x) T^{A} d x^{\mu}=\mathcal{A}^{A}(x) T^{A}$, the NAST for $S U(N)$ is given by

$$
\begin{aligned}
W^{C}[\mathcal{A}] & =\int[d \mu(\xi)]_{C} \exp \left(i g \oint_{C}\left[n^{A} \mathcal{A}^{A}+\frac{1}{g} \omega\right]\right) \\
& =\int[d \mu(\xi)]_{C} \exp \left(i g \int_{S: \partial S=C}\left[d\left(n^{A} \mathcal{A}^{A}\right)+\frac{1}{g} \Omega_{K}\right]\right),
\end{aligned}
$$

where we have defined

$$
\begin{aligned}
n^{A}(x) & :=\left\langle\Lambda\left|V(x) T^{A} V^{\dagger}(x)\right| \Lambda\right\rangle, \\
\omega(x) & :=\left\langle\Lambda\left|i V(x) d V^{\dagger}(x)\right| \Lambda\right\rangle=\left\langle\Lambda\left|i \xi^{\dagger}(x) d \xi(x)\right| \Lambda\right\rangle,
\end{aligned}
$$


and $\Omega_{K}$ is the Kähler two-form given by

$$
\Omega_{K}:=d \omega
$$

The NAST (5) implies that the Wilson loop is rewritten into

$$
W^{C}[\mathcal{A}]=\int[d \mu(\xi)]_{C} \exp \left(i g \oint_{C} a\right)=\int[d \mu(\xi)]_{C} \exp \left(i g \int_{S: C=\partial S} f\right) .
$$

First, $a$ is the connection one-form,

$$
a:=n^{A} \mathcal{A}^{A}+\frac{1}{g} \omega=\left\langle\Lambda\left|\mathcal{A}^{V}\right| \Lambda\right\rangle
$$

where $\mathcal{A}^{V}:=V \mathcal{A} V^{\dagger}+\frac{i}{g} V d V^{\dagger}$ is the gauge transformation of $\mathcal{A}$ by $V \in F_{N-1}$. For quark in the fundamental representation,

$$
a=\operatorname{tr}\left(\mathcal{H} \mathcal{A}^{V}\right)
$$

Therefore, the one-form $a$ is equal to the diagonal piece of the gauge-transformed potential $\mathcal{A}^{V}$. Next, $f$ is the curvature two-form,

$$
f:=d a=d C+\frac{1}{g} d \omega=d C+\frac{1}{g} \Omega_{K}
$$

where we defined the one-form, $C:=n^{A} \mathcal{A}^{A}$. The first piece $d C$ in $f$ does not contribute to the magnetic current, due to the Bianchi identity. On the other hand, the second term $\Omega_{K}$ in $f$ leads to the non-vanishing magnetic current,

$$
k_{\mu}:=\partial_{\nu}{ }^{*} f_{\mu \nu} \neq 0
$$

where ${ }^{*} f_{\mu \nu}$ is the Hodge dual of $f_{\mu \nu}$ in four dimensions, ${ }^{*} f_{\mu \nu}:=\frac{1}{2} \epsilon_{\mu \nu \rho \sigma} f_{\rho \sigma}$. In general, the (curvature) two-form $f=d\left(n^{A} \mathcal{A}^{A}\right)+\Omega_{K}$ in the NAST is the Abelian field strength (which is invariant even under the non-Abelian gauge transformation of $G=S U(N)$ ), i.e., the generalized 't Hooft-Polyakov tensor for $S U(N)$,

$$
f_{\mu \nu}(x):=\partial_{\mu}\left(n^{A}(x) \mathcal{A}_{\nu}^{A}(x)\right)-\partial_{\nu}\left(n^{A}(x) \mathcal{A}_{\mu}^{A}(x)\right)+\frac{i}{g} \mathbf{n}(x) \cdot\left[\partial_{\mu} \mathbf{n}(x), \partial_{\nu} \mathbf{n}(x)\right] .
$$

The invariance of $f$ is obvious from the NAST (9), since the L.H.S. of (9), i.e., $W^{C}[\mathcal{A}]$ is gauge invariant and the measure $[d \mu(\xi)]_{C}$ in the R.H.S. is also invariant under the $G$ gauge transformation. Otherwise, the R.H.S. is zero. In the case of fundamental representation, the invariance is easily seen, because it is possible to rewrite (14) into the manifestly gauge invariant form: $f_{\mu \nu}(x):=$ $\operatorname{tr}\left(\mathbf{n}(x) \mathcal{F}_{\mu \nu}(x)+\frac{i}{g} \mathbf{n}(x) \cdot\left[D_{\mu} \mathbf{n}(x), D_{\nu} \mathbf{n}(x)\right]\right)$, where $\mathcal{F}_{\mu \nu}(x):=\partial_{\mu} \mathcal{A}_{\nu}(x)-\partial_{\nu} \mathcal{A}_{\mu}(x)-$ $i g\left[\mathcal{A}_{\mu}(x), \mathcal{A}_{\nu}(x)\right]$, and $D_{\mu} \mathbf{n}(x):=\partial_{\mu} \mathbf{n}(x)-i g\left[\mathcal{A}_{\mu}(x), \mathbf{n}(x)\right]$. 


\section{ABELIAN DOMINANCE}

In our framework, the Abelian dominance and the monopole dominance are understood as implying the first and the second equality respectively,

$$
\left\langle W^{C}[\mathcal{A}]\right\rangle_{Y M} \cong\left\langle\exp \left(i g \oint_{C} a\right)\right\rangle_{A P E G T} \cong\left\langle\exp \left(i \oint_{C} \omega\right)\right\rangle_{A P E G T}
$$

where APEGT denotes the Abelian-projected effective gauge theory [3]. Numerical simulations show that the monopole part exhibits the area law and $\sigma_{\text {Abel }}$ exhausts the full string tension obtained from the non-Abelian Wilson loop (i.e., monopole dominance in the string tension or area law),

$$
\left\langle\exp \left(i \oint_{C} \omega\right)\right\rangle_{A P E G T} \sim \exp \left(-\sigma_{A b e l}|S|\right)
$$

while $\left\langle\exp \left(i g \oint_{C} a-i \oint_{C} \omega\right)\right\rangle_{A P E G T}$ does not exhibits the area law. This result implies that the area law of the original non-Abelian Wilson loop,

$$
\left\langle W^{C}[\mathcal{A}]\right\rangle_{Y M} \sim \exp (-\sigma|S|), \quad \sigma \cong \sigma_{\text {Abel }} .
$$

The monopole dominance in this sense was derived for $S U(2)$ in [6] by showing that the dominant contribution to the area law comes from the monopole piece alone, $\Omega_{K}=d \omega$. In [7], the monopole dominance and the area law of the Wilson loop have been shown based on the APEGT for $G=S U(2)$. Now this scenario can be extended into $G=S U(N)$.

\section{MAGNETIC MONOPOLES IN YANG-MILLS THEORY}

The existence of magnetic monopole is suggested from the non-trivial Homotopy groups $\pi_{2}(G / H)$. In the case (II), $\pi_{2}\left(F_{2}\right)=\pi_{2}(S U(3) /(U(1) \times U(1)))=\pi_{1}(U(1) \times$ $U(1))=\mathbf{Z}+\mathbf{Z}$. On the other hand, in the case (I), i.e., $[\mathrm{m}, 0]$ or $[0, \mathrm{n}] \pi_{2}\left(C P^{2}\right)=$ $\pi_{2}(S U(3) / U(2))=\pi_{1}(U(2))=\pi_{1}(S U(2) \times U(1))=\pi_{1}(U(1))=\mathbf{Z}$. Note that $C P^{n}$ NLSM has only the local $U(1)$ invariance for any $n$. It is this $\mathrm{U}(1)$ invariance that corresponds to a kind of Abelian magnetic monopole in the case (I). This magnetic monopole may be related to the non-Abelian magnetic monopole proposed by $\mathrm{E}$. Weinberg et al.

This situation should be compared with the $S U(2)$ case where the maximal stability group is always given by the maximal torus $H=U(1)$ irrespective of the representation. Therefore, the coset is given by $G / H=S U(2) / U(1)=F_{1}=$ $C P^{1} \cong S^{2} \cong S O(3)$ and $\pi_{2}(S U(2) / U(1))=\pi_{2}\left(F_{1}\right)=\pi_{2}\left(C P^{1}\right)=\mathbf{Z}$, for arbitrary representation. Actually, the NAST derived in this paper shows that the fundamental quark feels only the $U(1)$ embedded in the maximal stability group $U(2)$ as a magnetic monopole (This is a component along the highest-weight). 


\section{AREA LAW OF THE WILSON LOOP}

The (full) string tension $\sigma$ is defined by

$$
\sigma:=-\lim _{A(C) \rightarrow \infty} \frac{1}{A(C)} \ln \left\langle W^{C}[\mathcal{A}]\right\rangle_{Y M_{4}},
$$

where $A(C)$ is the minimal area spanned by the Wilson loop $C$.

We estimate the Wilson loop in the reformulation of the Yang-Mills theory which was proposed by the author and was called the perturbative deformation of a topological quantum field theory [4]. The Wilson loop is written as

$$
\left\langle W^{C}[\mathcal{A}]\right\rangle_{Y M_{4}}=\left\langle\left\langle\exp \left[i g \oint_{C} d x^{\mu} n^{A}(x) \mathcal{V}_{\mu}^{A}(x)\right]\right\rangle_{p Y M_{4}} \exp \left[i \oint_{C} \omega\right]\right\rangle_{T Q F T_{4}} .
$$

Here the expectation value $\langle(\cdots)\rangle_{p Y M}^{\mathcal{V}}$ for the field $\mathcal{V}$ is calculated in the perturbation theory in the coupling constant $g$. On the other hand, the expectation value $\langle(\cdots)\rangle_{T Q F T}^{U}$ should be calculated in a non-perturbative way to incorporate the topological contribution where $U$ is a compact gauge variable,

$$
S_{T Q F T}[\Omega, C, \bar{C}, B]:=\int_{\mathbf{R}^{4}} d^{4} x i \delta_{B} \bar{\delta}_{B} \operatorname{tr}_{G / H}\left[\frac{1}{2} \Omega_{\mu}(x) \Omega_{\mu}(x)+i C(x) \bar{C}(x)\right],
$$

where $\Omega_{\mu}(x):=\frac{i}{g} U(x) \partial_{\mu} U^{\dagger}(x)$. This reformulation leads to the result:

$$
\left\langle W^{C}[\mathcal{A}]\right\rangle_{Y M_{4}}=\left\langle\exp \left[i \oint_{C} \omega\right]\right\rangle_{T Q F T_{4}}\left[1+O\left(g^{2}\right)\right] .
$$

This implies the magnetic monopole dominance in the area law of the Wilson loop.

For the planar Wilson loop $C$, the Parisi-Soulous dimensional reduction [4] leads to

$$
\left\langle\exp \left[i \oint_{C} \omega\right]\right\rangle_{T Q F T_{4}}=\left\langle\exp \left[i \oint_{C} \omega\right]\right\rangle_{N L S M_{2}},
$$

where the two-dimensional nonlinear sigma model (NLSM) has the action,

$$
S_{N L S M}[U, C, \bar{C}]:=2 \pi \int_{\mathbf{R}^{2}} d^{2} x \operatorname{tr}_{G / H}\left[\frac{1}{2} \Omega_{\mu}(x) \Omega_{\mu}(x)+i C(x) \bar{C}(x)\right] .
$$

By making use of the complex coordinates of the flag space $G / H$, the action is rewritten as

$$
\begin{aligned}
S_{N L S M} & =\frac{\pi}{g^{2}} \int_{\mathbf{R}^{2}} d^{2} x g_{\alpha \bar{\beta}} \frac{\partial w^{\alpha}}{\partial x_{a}} \frac{\partial \bar{w}^{\beta}}{\partial x_{a}} \quad(a=1,2) \\
& =\frac{\pi}{g^{2}} \int_{\mathbf{C}} d z d \bar{z} g_{\alpha \bar{\beta}}\left(\frac{\partial w^{\alpha}}{\partial z} \frac{\partial \bar{w}^{\beta}}{\partial \bar{z}}+\frac{\partial w^{\alpha}}{\partial \bar{z}} \frac{\partial \bar{w}^{\beta}}{\partial z}\right),
\end{aligned}
$$


where $z=x+i y=x_{1}+i x_{2} \in \mathbf{C} \cong \mathbf{R}^{2}$, and $d x d y=d x_{1} d x_{2}=\frac{i}{2} d z d \bar{z}$, and we have omitted the ghost term, $C(x) \bar{C}(x)$. Here $g(\mu)$ is the running Yang-Mills coupling constant whose running is given by the perturbative deformation in fourdimensional Yang-Mills theory. For the quark in the fundamental representation of $S U(N)$, the relevant NLSM is given by $C P^{N-1}$ model. We can show the area law,

$$
\left\langle\exp \left[i \oint_{C} \omega\right]\right\rangle_{C P_{2}^{N-1}} \sim \exp \left(-\sigma_{0} T R\right),
$$

by the instanton calculus (dilute instanton-gas approximation) or by the large $N$ expansion in the two-dimensional $C P^{N-1}$ model, see [10].

In summary, we have given a new derivation of non-Abelian Stokes theorem for $G=S U(N)$ for $N \geq 2$ which reduces to the previous result for $S U(2)$. This version of non-Abelian Wilson loop is very helpful to see the role played by the magnetic monopole in the calculation of the non-Abelian Wilson loop. Combining this non-Abelian Stokes theorem with the Abelian-projected effective gauge theory for $S U(N)$, we have explained the Abelian dominance for the Wilson loop in $S U(N)$ Yang-Mills gauge theory. For $S U(N)$ with $N$ greater than two, we must distinguish the maximal stability group $\tilde{H}$ and the residual gauge group $H=U(1)^{N-1}$.

By making use of the non-Abelian Stokes theorem in a novel reformulation of the Yang-Mills theory proposed by one of the authors [4], the derivation of the area law of the non-Abelian Wilson loop in four-dimensional Yang-Mills theory has been reduced to the two-dimensional problem of calculating the expectation value of the Abelian Wilson loop in the coset $G / H$ non-linear sigma model. Especially, in order to show confinement of the fundamental quark in four-dimensional $S U(N)$ Yang-Mills theory in the MA gauge, we have only to consider the two-dimensional $C P^{N-1}$ model. The details will be given in $[9,10]$. A Monte Carlo simulation on a lattice will be efficient to confirm the above picture of quark confinement.

\section{REFERENCES}

1. Nambu, Y., Phys. Rev. D 10, 4262 (1974).

't Hooft, G., in: High Energy Physics, edited by A. Zichichi (Editorice Compositori, Bologna, 1975).

Mandelstam, S., Phys. Report 23, 245 (1976).

2. 't Hooft, G., Nucl. Phys. B 190 [FS3], 455 (1981).

3. Kondo, K.-I., hep-th/9709109, Phys. Rev. D 57, 7467 (1998).

4. Kondo, K.-I., hep-th/9801024, Phys. Rev. D 58, 105019 (1998).

5. Kondo, K.-I., hep-th/9803133, Phys. Rev. D 58, 085013 (1998).

6. Kondo, K.-I., hep-th/9805153, Phys. Rev. D 58, 105016 (1998).

7. Kondo, K.-I., hep-th/9810167, Phys. Lett. B 455, 251 (1999).

8. Kondo, K.-I., hep-th/9904045.

9. Kondo, K.-I., and Taira, Y., hep-th/9906129.

10. Kondo, K.-I., and Taira, Y., in preparation. 